PROCEEDINGS OF THE

AMERICAN MATHEMATICAL SOCIETY

Volume 131, Number 6, Pages 1695-1704

S 0002-9939(02)06735-7

Article electronically published on October 1, 2002

\title{
RATIONAL VERSUS REAL COHOMOLOGY ALGEBRAS OF LOW-DIMENSIONAL TORIC VARIETIES
}

\author{
EVA MARIA FEICHTNER
}

(Communicated by John R. Stembridge)

\begin{abstract}
We show that the real cohomology algebra of a compact toric variety of complex dimension 2 is determined, up to isomorphism, by the combinatorial data of its defining fan. Surprisingly enough, this is no longer the case when taking rational coefficients. Moreover, we show that neither the rational nor the real or complex cohomology algebras of compact quasi-smooth toric varieties are combinatorial invariants in general.
\end{abstract}

\section{INTRODUCTION}

The interest in toric varieties from various fields of mathematics is widely due to their two-fold nature: On one hand, they are algebro-geometric objects; on the other hand, they are defined through discrete geometric objects and possess natural combinatorial data. Much as algebro-geometric properties of toric varieties have combinatorial characterizations, one is led to ask whether topological invariants of a toric variety are determined by the combinatorics of the defining fan, i.e., whether, as a matter of fact, they are combinatorial invariants of the fan. In the present paper we address this question with regard to the rational cohomology algebras of compact quasi-smooth toric varieties.

On the discrete-geometric side, we will be concerned with complete fans of strictly convex, simplicial cones in $\mathbb{R}^{d}$. Let $\Sigma$ be such a fan, generated by primitive lattice vectors $v_{1}, \ldots, v_{n}$ in $N \cong \mathbb{Z}^{d}$. Denote by $\Delta(\Sigma)$ the simplicial complex defined on the index set of generating lattice vectors, with a subset forming a simplex in $\Delta(\Sigma)$ if and only if the corresponding lattice vectors span a cone in $\Sigma$. We refer to the abstract simplicial complex $\Delta(\Sigma)$ as the combinatorial data of $\Sigma$. Equally, we could refer to the face poset of $\Sigma$. Asking whether a topological invariant is in fact a combinatorial invariant thus means asking whether the invariant only depends on $\Delta(\Sigma)$.

Complete simplicial fans define toric varieties that are compact and quasismooth, the latter meaning that at worst they have finite quotient singularities. For

Received by the editors May 14, 1999 and, in revised form, December 18, 2001, January 25, 2002, and January 30, 2002.

2000 Mathematics Subject Classification. Primary 14M25; Secondary 14F25, 52B20.

Key words and phrases. Toric varieties, cohomology algebras, simplicial fans, Hodge-RiemannMinkowski inequalities. 
the basic theory of toric varieties we refer to the standard textbooks by Oda, Fulton, or Ewald $[\mathrm{Od}, \mathrm{Fu}, \mathrm{Ew}$, as well as to the classical survey paper by Danilov [Da].

For a compact quasi-smooth toric variety the cohomology groups with rational coefficients are determined by the combinatorial data of the defining fan. Together with a description of the ring structure due to Danilov [Da], this makes compact quasi-smooth toric varieties a natural class for our investigations. Let us mention here that the integer cohomology of toric varieties is not even combinatorially determined in complex dimension $2[\mathrm{FY}]$, nor are the rational Betti numbers combinatorial invariants beyond the quasi-smooth case [McC].

Let us sketch the contents of this paper: In Section 2 we state Danilov's result (Theorem 2.1) along with a discussion of some geometric aspects of his algebra presentation.

Section 3 is devoted to the situation in complex dimension 2 which turns out to be surprisingly intricate: We show that the real cohomology algebra of a compact toric variety of complex dimension 2 is completely determined by the combinatorial data of its defining fan, i.e., by the number of rays alone (Theorem 3.7). We obtain this result as a consequence of certain real symmetric bilinear forms on cohomology algebras of projective quasi-smooth toric varieties being combinatorially determined (Theorem [3.6). This fact, in turn, is the algebro-geometric counterpart of the Hodge-Riemann-Minkowski inequalities on weight spaces of simple polytopes $\mathrm{McM}$ Thm. 8.2]. Here we give a proof of Theorem 3.6 from the algebro-geometric viewpoint. In contrast to our result on the real cohomology algebra, we show that the rational cohomology algebra of a compact toric variety in complex dimension 2 is no longer a combinatorial invariant (Example 3.8).

In Section 4 we present an example of toric varieties in complex dimension 3 which shows that neither the rational nor the real or the complex cohomology algebras of compact quasi-smooth toric varieties are combinatorial invariants in general (Example 4.1).

Contrasting our results, let us mention that the rational equivariant cohomology algebra of a compact quasi-smooth toric variety $X_{\Sigma}$ with its natural torus action is combinatorially determined: it is isomorphic to the Stanley-Reisner ring of the simplicial complex $\Delta(\Sigma)([\mathrm{Br}$, Remark p. 17] and $[\mathrm{Bi}])$.

\section{Preliminaries on Danilov's algebra presentation}

We first state a presentation for the rational cohomology algebra of a compact quasi-smooth toric variety due to Danilov [Da], undoubtedly, a fundamental result on the cohomology ring structure of toric varieties. Along with summarizing some of the algebraic-topological properties of the varieties in question, we recall the geometric meaning of the multiplicative generators (compare [Da, §5.7, §10]), and subsequently derive a description of the cohomological dual of the fundamental class for further reference.

Theorem 2.1 ([Da, $\S 10])$. Let $\Sigma$ be a complete simplicial fan in $\mathbb{R}^{d}$, generated by primitive lattice vectors $v_{1}, \ldots, v_{n}$ in $N \cong \mathbb{Z}^{d}$ with the coordinates of $v_{i}$ denoted by $v_{i}^{(1)}, \ldots, v_{i}^{(d)}$ for $i=1, \ldots, n$. Let $f_{i}(\Sigma)$ denote the number of $i$-dimensional simplices in $\Delta(\Sigma), i=0, \ldots, d-1$, and set $f_{-1}(\Sigma):=1$. 
The rational cohomology algebra of the associated toric variety $X_{\Sigma}$ has the following presentation in terms of generators and relations:

$$
H^{*}\left(X_{\Sigma}, \mathbb{Q}\right) \cong \mathbb{Q}\left[z_{1}, \ldots, z_{n}\right] /\left\langle\begin{array}{ll}
\prod_{j=1}^{k} z_{i_{j}} & \text { for }\left\{i_{1}, \ldots, i_{k}\right\} \notin \Delta(\Sigma), \\
\sum_{i=1}^{n} v_{i}^{(t)} z_{i} & \text { for } 1 \leq t \leq d
\end{array}\right\rangle .
$$

The rational Betti numbers are determined by the face numbers of $\Sigma$ :

$$
\operatorname{dim}_{\mathbb{Q}} H^{2 k}\left(X_{\Sigma}, \mathbb{Q}\right)=\sum_{i=0}^{k}(-1)^{k-i}\left(\begin{array}{l}
d-i \\
d-k
\end{array}\right) f_{i-1}(\Sigma), \quad 0 \leq k \leq d .
$$

Remark 2.2. Presentations for the real and complex cohomology algebras are obtained by tensoring with $\mathbb{R}$ or $\mathbb{C}$, respectively. In case $X_{\Sigma}$ is smooth, i.e., the defining fan is unimodular, the above presentation holds with coefficients in $\mathbb{Z}$. Let us further point out that the rational Betti numbers coincide with the entries of the $h$-vector of $\Delta(\Sigma)$ (compare [Zi, Def. 8.18]).

Any toric variety $X_{\Sigma}$ is endowed with a natural torus action, where the orbits are in one-to-one correspondence with the cones in the defining fan $\Sigma \subseteq \mathbb{R}^{d}$. The orbit closures $F_{S}$ for $S \in \Delta(\Sigma)$ are toric subvarieties of $X_{\Sigma}$ with combinatorial data given by the star of $S$ in $\Delta(\Sigma), \operatorname{st}(S)=\{T \in \Delta(\Sigma) \mid S \subseteq T\}$. In particular, $\operatorname{dim}_{\mathbb{C}} F_{S}=d-1-\operatorname{dim} S$. In case $\Sigma$ is a complete simplicial fan, the associated algebraic cycles $\left[F_{S}\right], S \in \Delta(\Sigma)$, generate the homology of $X_{\Sigma}$ Da, Prop. 10.3].

A compact quasi-smooth toric variety $X_{\Sigma}$ is a rational homology manifold [Da, §14]. In particular, this allows for a canonical homology generator in top dimension, the fundamental class $\left[X_{\Sigma}\right] \in H_{2 d}\left(X_{\Sigma}, \mathbb{Q}\right)$ : It is the unique class that restricts to the local (canonical) orientation class in $H_{2 d}\left(X_{\Sigma}, X_{\Sigma} \backslash\{x\}, \mathbb{Q}\right.$ ) for every $x \in X_{\Sigma}$. Moreover, all rational intersection homology groups are isomorphic, and the canonical maps connecting cohomology, resp. homology, groups with intersection homology are isomorphisms $[\mathrm{Fu}$, p. 105]:

$$
H^{2 d-k}\left(X_{\Sigma}, \mathbb{Q}\right) \stackrel{\cong}{\longrightarrow} I H_{k}\left(X_{\Sigma}, \mathbb{Q}\right) \stackrel{\cong}{\longrightarrow} H_{k}\left(X_{\Sigma}, \mathbb{Q}\right) \quad \text { for } 0 \leq k \leq 2 d .
$$

This composition of maps factors the Poincaré duality map

$$
\phi=-\cap\left[X_{\Sigma}\right]: \quad H^{2 d-k}\left(X_{\Sigma}, \mathbb{Q}\right) \longrightarrow H_{k}\left(X_{\Sigma}, \mathbb{Q}\right) \quad \text { for } 0 \leq k \leq 2 d,
$$

given by cap product multiplication of cohomology classes on $X_{\Sigma}$ with the fundamental class $\left[X_{\Sigma}\right]$ GM, §1.4].

The Poincaré duality isomorphism allows us to relate the algebraic cycles $\left[F_{S}\right]$, $S \in \Delta(\Sigma)$, to the cohomology presentation stated in Theorem 2.1 the Poincaré duals $\left[F_{\{i\}}\right]^{\vee}:=\phi^{-1}\left(\left[F_{\{i\}}\right]\right)$ of homology classes represented by the divisors $F_{\{i\}}$, $\{i\} \in \Delta_{0}(\Sigma)$, generate the rational cohomology algebra of $X_{\Sigma}$. In fact, they correspond to the abstract algebra generators in the Danilov presentation.

Multiplication among these generators - in the dual picture: intersection of the divisors - is described by

$$
\begin{aligned}
& {\left[F_{\left\{i_{1}\right\}}\right]^{\vee} } \smile \ldots \smile\left[F_{\left\{i_{k}\right\}}\right]^{\vee} \\
& r=\left\{\begin{aligned}
\frac{1}{\operatorname{mult}(\sigma(S) ; N)}\left[F_{S}\right]^{\vee} & \text { for }\left\{i_{1}, \ldots, i_{k}\right\}=S \in \Delta(\Sigma), \\
0 & \text { otherwise, }
\end{aligned}\right.
\end{aligned}
$$


where $\left[F_{S}\right]^{\vee}:=\phi^{-1}\left(\left[F_{S}\right]\right), \sigma(S)$ is the cone in $\Sigma$ corresponding to $S \in \Delta(\Sigma)$, and $\operatorname{mult}(\sigma ; N)$, the multiplicity of $\sigma$, is defined as the index

$$
\operatorname{mult}(\sigma ; N):=\left|N \cap(\sigma-\sigma):\left\langle v_{i_{1}}, \ldots, v_{i_{k}}\right\rangle\right|
$$

of the subgroup generated by the primitive generating vectors $v_{i_{1}}, \ldots, v_{i_{k}}$ of $\sigma$ in the lattice $N$ restricted to the linear hull of $\sigma$. Equivalently, $\operatorname{mult}(\sigma ; N)$ is the number of lattice points in the parallelotope $P_{\sigma}:=\left\{\sum_{j=1}^{k} \alpha_{j} v_{i_{j}} \mid 0 \leq \alpha_{j}<1\right\}$. The multiplicity is a measure of how far the generating vectors of a simplicial cone $\sigma$ are from being part of a lattice basis for $N$, i.e., how far $\sigma$ is from being unimodular.

Proposition 2.3. Let $X_{\Sigma}$ be a compact quasi-smooth toric variety with defining fan $\Sigma \subseteq \mathbb{R}^{d}$ as in Theorem 2.1. Then the cohomological dual of the fundamental class of $X_{\Sigma}$ can be written as

$$
\left[X_{\Sigma}\right]^{*}=\operatorname{det}\left(v_{i_{1}}, \ldots, v_{i_{d}}\right) \prod_{j=1}^{d} z_{i_{j}} \quad \text { for any }\left\{i_{1}, \ldots, i_{d}\right\} \in \Delta_{d-1}(\Sigma),
$$

where the $z_{i_{j}}$ denote generators in the Danilov presentation.

Proof. For $S \in \Delta_{d-1}(\Sigma)$ representing a top-dimensional cone in $\Sigma$ the subvariety $F_{S}$ is a single point in $X_{\Sigma}$. The Poincaré dual $\left[F_{S}\right]^{\vee}$ of the algebraic cycle $\left[F_{S}\right] \in$ $H_{0}\left(X_{\Sigma}\right), S \in \Delta_{d-1}(\Sigma)$, evaluates to 1 on the fundamental class $\left[X_{\Sigma}\right]$ :

$$
\left\langle\left[F_{S}\right]^{\vee},\left[X_{\Sigma}\right]\right\rangle=\epsilon\left(\left[F_{S}\right]^{\vee} \cap\left[X_{\Sigma}\right]\right) \stackrel{(1)}{=} \epsilon\left(\left[F_{S}\right]\right)=1,
$$

where $\epsilon$ denotes the augmentation map taking points to 1, and (1) is a consequence of the explicit description of the Poincaré duality isomorphism stated above. Hence

$$
\left[X_{\Sigma}\right]^{*}=\left[F_{S}\right]^{\vee} \quad \text { for any } S \in \Delta_{d-1}(\Sigma),
$$

and the stated description follows from the multiplication rule $(*)$ for generators in the Danilov presentation.

\section{Cohomology of toric VARIETIES OF COMPlex Dimension 2}

In this section we consider compact toric varieties associated with complete fans in $\mathbb{R}^{2}$. Such fans are per se simplicial and strongly polytopal, hence the associated toric varieties are quasi-smooth and projective.

Let $\Sigma$ denote a complete fan in $\mathbb{R}^{2}$, spanned by $n$ primitive lattice vectors $v_{1}, \ldots, v_{n}$ in $N \cong \mathbb{Z}^{2}$. According to Theorem 2.1 the cohomology of the associated toric variety $X_{\Sigma}$ with coefficients in $\mathbb{K}, \mathbb{K}=\mathbb{Q}, \mathbb{R}$ or $\mathbb{C}$ is linearly isomorphic to a graded vector space $U$ over $\mathbb{K}$ with total dimension $n$ :

$$
\operatorname{dim}_{\mathbb{K}} U_{i}= \begin{cases}1 & \text { for } i=0,4 \\ n-2 & \text { for } i=2, \\ 0 & \text { otherwise }\end{cases}
$$

The structure of an associative graded-commutative algebra on $U$ is fully described by specifying a symmetric bilinear map $\circ: U_{2} \times U_{2} \longrightarrow U_{4}$ that describes multiplication among elements of degree 2 . Fix a linear basis $\mathcal{B}$ for $U$, say $\mathcal{B}_{0}=\{1\}$ for $U_{0}$, 
$\mathcal{B}_{2}=\left\{u_{1}, \ldots, u_{n-2}\right\}$ for $U_{2}$, and $\mathcal{B}_{4}=\{u\}$ for $U_{4}$. We encode the algebra structure on $U$ determined by $\circ: U_{2} \times U_{2} \longrightarrow U_{4}$ into a matrix over $\mathbb{K}$ :

$$
M_{\mathcal{B}}^{(U, \circ)}=\left(\alpha_{i, j}\right)_{1 \leq i, j \leq n-2},
$$

with $\alpha_{i, j} \in \mathbb{K}$ defined by $u_{i} \circ u_{j}=\alpha_{i, j} u$.

Proposition 3.1. Symmetric bilinear maps $\circ_{1}, \circ_{2}: U_{2} \times U_{2} \longrightarrow U_{4}$ yield isomorphic algebra structures on $U$ if and only if

$$
M_{\mathcal{B}}^{\left(U, \circ_{1}\right)}=q I_{n-2} Q M_{\mathcal{B}}^{\left(U, \circ_{2}\right)} Q^{T}
$$

for some $q \in \mathbb{K}^{*}, Q \in \mathrm{GL}(n-2, \mathbb{K})$, where $I_{n-2}$ denotes the identity in $\mathrm{GL}(n-2, \mathbb{K})$.

Proof. Let $\varphi:\left(U, \circ_{1}\right) \longrightarrow\left(U, \circ_{2}\right)$ be an isomorphism of algebras. Then

$$
M_{\mathcal{B}}^{\left(U, \circ_{1}\right)}=M_{\varphi(\mathcal{B})}^{\left(U, o_{2}\right)} .
$$

Encoding $\left(U, \circ_{2}\right)$ with respect to $\mathcal{B}$ requires basis changes by $Q:=\varphi_{\left.\right|_{U_{2}}} \in \mathrm{GL}(n-2, \mathbb{K})$ in degree 2 , and by $\frac{1}{q} \in \mathbb{K}^{*}$ - a scalar multiplication - in degree 4 , where $q$ is defined by $\varphi(u)=q \cdot u$.

Conversely, if matrices that encode algebra structures $\left(U, \circ_{1}\right)$ and $\left(U, \circ_{2}\right)$, respectively, are related as described in the proposition, then $\varphi:\left(U, \circ_{1}\right) \longrightarrow\left(U, \circ_{2}\right)$ defined by $\varphi\left(u_{i}\right)=Q u_{i}, i=1, \ldots, n-2$, and $\varphi(u)=\frac{1}{q} \cdot u$ is an algebra isomorphism.

We introduce an invariant of the toric varieties under consideration that is closely related, but finer than the abstract isomorphism type of their cohomology algebras:

Definition 3.2. Let $X_{\Sigma}$ be a compact toric variety of complex dimension 2 . We associate a symmetric bilinear form $\xi(\Sigma): H^{2}\left(X_{\Sigma}, \mathbb{K}\right) \otimes H^{2}\left(X_{\Sigma}, \mathbb{K}\right) \longrightarrow \mathbb{K}$ by defining

$$
\xi(\Sigma)(a, b)=\left\langle a \smile b,\left[X_{\Sigma}\right]\right\rangle \quad \text { for } a, b \in H^{2}\left(X_{\Sigma}, \mathbb{K}\right),
$$

where $\left\langle\cdot,\left[X_{\Sigma}\right]\right\rangle$ denotes evaluation on the fundamental class of $X_{\Sigma}$.

With Proposition 2.3 at hand we are able to write out explicit matrix descriptions for the symmetric bilinear forms $\xi(\Sigma)$. In the linear basis for $H^{2}\left(X_{\Sigma}, \mathbb{K}\right)$ given by the generators $z_{1}, \ldots, z_{n-2}$ of the Danilov presentation, the matrix description reads

$$
M(\xi(\Sigma))=\left(\beta_{i, j}\right)_{1 \leq i, j \leq n-2}
$$

with $\beta_{i, j} \in \mathbb{K}$ defined by $z_{i} \smile z_{j}=\beta_{i, j} \cdot\left[X_{\Sigma}\right]^{*}$.

Using our characterization of isomorphisms of algebra structures on the graded vector space $U \cong H^{*}\left(X_{\Sigma}, \mathbb{K}\right)$ (Proposition 3.1) we can state the following observation:

Proposition 3.3. Let $X_{\Sigma}$ and $X_{\Sigma^{\prime}}$ be compact toric varieties of complex dimension 2. If the symmetric bilinear forms $\xi(\Sigma)$ and $\xi\left(\Sigma^{\prime}\right)$ are equivalent over $\mathbb{K}$, then the cohomology algebras of the varieties with coefficients in $\mathbb{K}$ are isomorphic.

The converse however is not true:

Example 3.4. Consider the complete fans $\Sigma$ and $\Sigma^{\prime}$ in $\mathbb{R}^{2}$, where $\Sigma$ is spanned by $v_{1}=(1,0), v_{2}=(0,1)$, and $v_{3}=(-1,-1)$, and $\Sigma^{\prime}$ is spanned by $v_{1}^{\prime}=(1,0), v_{2}^{\prime}=$ $(0,1)$, and $v_{3}^{\prime}=(-2,-1)$. The Danilov presentations for the rational cohomology algebras of the toric varieties $X_{\Sigma}$ and $X_{\Sigma^{\prime}}$ both reduce to $\mathbb{Q}[z] /\left\langle z^{3}\right\rangle$, where $z$ corresponds to $\left[F_{\{3\}}\right]^{\vee}$ in $H^{2}(X, \mathbb{Q})$. Descriptions of the cohomological duals of the 
fundamental classes in terms of $z$ however read: $\left[X_{\Sigma}\right]^{*}=\left[F_{\{2,3\}_{\Sigma}}\right]^{\vee}=z^{2}$ and $\left[X_{\Sigma^{\prime}}\right]^{*}=\left[F_{\{2,3\}_{\Sigma^{\prime}}}\right]^{\vee}=2 z^{2}$. Hence, $M(\xi(\Sigma))=(1)$, whereas $M\left(\xi\left(\Sigma^{\prime}\right)\right)=(1 / 2)$, and thus the symmetric bilinear forms $\xi(\Sigma)$ and $\xi\left(\Sigma^{\prime}\right)$ are non-equivalent over $\mathbb{Q}$.

The forms $\xi(\Sigma)$ belong to a larger family of bilinear forms defined on the cohomology of any compact complex projective quasi-smooth variety. Recall that the Hard Lefschetz Theorem holds for such varieties, i.e., iterated cup product multiplication with the cohomology class of a hyperplane section, $\omega \in H^{2}(X, \mathbb{K})$, gives isomorphisms on the graded pieces of $H^{*}(X, \mathbb{K})$ :

$$
H^{k}(X, \mathbb{K}) \stackrel{\smile \omega^{d-k}}{\longrightarrow} H^{2 d-k}(X, \mathbb{K}) \quad \text { for } 0 \leq k \leq d=\operatorname{dim}_{\mathbb{C}} X .
$$

For a detailed formulation of the Hard Lefschetz Theorem in the toric case see Od, Sect. 3.3, p. 135]; the general result is a consequence of work by Saito [Sa].

Definition 3.5. Let $X$ be a compact complex projective quasi-smooth variety of complex dimension $d$. Define bilinear forms $\xi_{k}: H^{k}(X, \mathbb{K}) \otimes H^{k}(X, \mathbb{K}) \longrightarrow \mathbb{K}$, $0 \leq k \leq d$, by

$$
\xi_{k}(a, b)=\left\langle a \smile b \smile \omega^{d-k},[X]\right\rangle \quad \text { for } a, b \in H^{k}(X, \mathbb{K}),
$$

where $\omega \in H^{2}(X, \mathbb{K})$ is the "Lefschetz element" of (3.1) and $\langle\cdot,[X]\rangle$ denotes evaluation on the fundamental class.

Theorem 3.6. Let $X_{\Sigma}$ be a compact projective quasi-smooth toric variety with complete defining fan $\Sigma \subseteq \mathbb{R}^{d}$. Then the real symmetric bilinear forms $\xi_{2 k}$ on $H^{2 k}\left(X_{\Sigma}, \mathbb{R}\right), 0 \leq k \leq\left\lfloor\frac{d}{2}\right\rfloor$, are determined up to equivalence by the combinatorial data of $\Sigma: \xi_{2 k}$ is non-degenerate and $(-1)^{k} \xi_{2 k}$ has $\sum_{i=0}^{k}(-1)^{i} h_{k-i}(\Sigma)$ positive and $\sum_{i=0}^{k-1}(-1)^{i} h_{k-i-1}(\Sigma)$ negative eigenvalues, with $h_{j}(\Sigma)$ denoting the $h$-vector entries of $\Delta(\Sigma)$.

Taking into account the isomorphism between real cohomology algebras of compact projective quasi-smooth toric varieties and certain subalgebras of the polytope algebra [McM, Thm.14.1], our result translates into a result by P. McMullen [McM] Sect. 8], the so-called Hodge-Riemann-Minkowski inequalities on weight spaces of simple polytopes. Our theorem complements McMullen's result from an algebrogeometric viewpoint: Due to de Rham and Hodge theory extending to the varieties in question [Da $\S 4, \S 12]$, we obtain Theorem [3.6 as a consequence of the classical Hodge-Riemann bilinear relations [GH. p. 123], and we explain why they take a particularly simple form in the toric case.

Proof of Theorem 3.6. As a consequence of the Hard Lefschetz Theorem for compact projective quasi-smooth toric varieties, there is a direct sum decomposition on each graded piece of the cohomology algebra, the so-called Lefschetz decomposition:

$$
H^{2 k}\left(X_{\Sigma}, \mathbb{K}\right) \cong \bigoplus_{j \geq 0} \omega^{j} P^{2 k-2 j}\left(X_{\Sigma}, \mathbb{K}\right) \quad \text { for } 0 \leq k \leq d,
$$

where

$$
P^{2 r}\left(X_{\Sigma}, \mathbb{K}\right)=\operatorname{Ker}\left(H^{2 r}\left(X_{\Sigma}, \mathbb{K}\right) \stackrel{\smile \omega^{d-2 r+1}}{\longrightarrow} H^{2 d-2 r+2}\left(X_{\Sigma}, \mathbb{K}\right)\right)
$$

is the primitive cohomology of $X_{\Sigma}$ in degree $2 r, 0 \leq r \leq\left\lfloor\frac{d}{2}\right\rfloor$.

It is easy to see that the Lefschetz decomposition of $H^{2 k}\left(X_{\Sigma}, \mathbb{K}\right)$ is orthogonal with respect to $\xi_{2 k}$. Moreover, $\xi_{2 k}(a, b)=\xi_{2 k-2 j}\left(a^{\prime}, b^{\prime}\right)$ for $a=\omega^{j} a^{\prime}, b=\omega^{j} b^{\prime} \in$ 
$\omega^{j} P^{2 k-2 j}\left(X_{\Sigma}\right)$, and thus it suffices to study the symmetric bilinear forms $\xi_{2 k}$ on the primitive part of $H^{*}\left(X_{\Sigma}, \mathbb{K}\right)$.

The real symmetric bilinear forms $\xi_{2 k}$ on $H^{*}\left(X_{\Sigma}, \mathbb{R}\right)$ are induced by the corresponding forms on $H^{*}\left(X_{\Sigma}, \mathbb{C}\right.$ ). The Hodge-Riemann bilinear relations (cf. $\mathrm{GH}$, p. 123]), a consequence of the Hard Lefschetz Theorem and of Hodge theory for the varieties in question, ensure that the forms are non-degenerate. Moreover, in even degrees the relations specify the number of positive and negative eigenvalues on the primitive cohomology referring to the Hodge filtration on $P^{*}\left(X_{\Sigma}, \mathbb{C}\right)$ :

$$
\sqrt{-1}^{p-q}(-1)^{(p+q)(p+q-1) / 2} \xi_{2 k}(x, \bar{x})>0,
$$

for $x \in P^{p+q}\left(X_{\Sigma}, \mathbb{C}\right) \cap H^{2 k}\left(X_{\Sigma}, \mathbb{R}\right)$.

Whereas all before-mentioned arguments hold in the generality of compact complex projective quasi-smooth varieties, we now need to use a particular feature of Hodge filtrations in the toric case: There is a spectral sequence converging to $H^{*}\left(X_{\Sigma}, \mathbb{C}\right)$,

$$
E_{1}^{p, q}=H^{q}\left(X_{\Sigma}, \Omega_{X_{\Sigma}}^{p}\right) \Rightarrow H^{p+q}\left(X_{\Sigma}, \mathbb{C}\right),
$$

with the $E_{1}$-term the cohomology of sheaves of differential forms on $X_{\Sigma}$. The spectral sequence collapses in its first term and yields the Hodge filtration on $H^{*}\left(X_{\Sigma}, \mathbb{C}\right)[\mathrm{Da}$, Thm. 12.5]. From [Da, Prop. 12.11] we see that for quasi-smooth toric varieties the tableau entries $H^{q}\left(X_{\Sigma}, \Omega_{X_{\Sigma}}^{p}\right)$ are trivial unless $p=q$, thus we conclude that the only non-trivial summand in the Hodge decomposition of $H^{2 k}\left(X_{\Sigma}, \mathbb{C}\right)$ is the summand of symmetric degree $(k, k)$.

The Hodge-Riemann bilinear relations (3.3) thus assert that the symmetric bilinear forms $\xi_{2 k}$ on $H^{2 k}\left(X_{\Sigma}, \mathbb{R}\right), 0 \leq k \leq\left\lfloor\frac{d}{2}\right\rfloor$, either have positive or negative eigenvalues on entire summands of the Lefschetz decomposition (3.2) and that the signs of eigenvalues alternate with $k$.

To determine the number of positive and negative eigenvalues observe that as a consequence of the Hard Lefschetz Theorem

$\operatorname{dim}_{\mathbb{R}} P^{2 k}\left(X_{\Sigma}, \mathbb{R}\right)=\operatorname{dim}_{\mathbb{R}} H^{2 k}\left(X_{\Sigma}, \mathbb{R}\right)-\operatorname{dim}_{\mathbb{R}} H^{2 k-2}\left(X_{\Sigma}, \mathbb{R}\right)=h_{k}(\Sigma)-h_{k-1}(\Sigma)$

for $0 \leq k \leq\left\lfloor\frac{d}{2}\right\rfloor$. The statement of the theorem thus follows.

Our main result on the description of real (and thus of complex) cohomology algebras of compact toric varieties in complex dimension 2 is now an easy consequence:

Theorem 3.7. The real cohomology algebra of a compact toric variety of complex dimension 2 is completely determined by the combinatorial data of its defining fan, i.e., by the number of rays alone. Let $\Sigma$ denote a complete fan in $\mathbb{R}^{2}$ as described above. Then

$$
H^{*}\left(X_{\Sigma}, \mathbb{R}\right) \cong \mathbb{R}\left[z_{1}, \ldots, z_{n-2}\right] /\left\langle\begin{array}{ll}
z_{1}^{2}+z_{i}^{2} & \text { for } 2 \leq i \leq n-2, \\
z_{i} z_{j} & \text { for } 1 \leq i<j \leq n-2
\end{array}\right\rangle .
$$

Proof. Theorem [3.6 asserts that for a fixed number of rays in $\Sigma$ the symmetric bilinear forms $\xi(\Sigma)=\xi_{2}$ are all equivalent over $\mathbb{R}$. Using Proposition 3.3 this implies that the real cohomology algebras are all of the same isomorphism type. In particular, $\xi(\Sigma)$ has $h_{0}(\Sigma)=1$ positive eigenvalue and $h_{1}(\Sigma)-h_{0}(\Sigma)=n-3$ negative eigenvalues. The explicit algebra presentation for $H^{*}\left(X_{\Sigma}, \mathbb{R}\right)$ follows. 
Theorem 3.7 does not extend to cohomology algebras with rational coefficients:

Example 3.8. Let $\Sigma$ denote the complete fan spanned by

$$
v_{1}=(1,0), \quad v_{2}=(0,1), \quad v_{3}=(-1,0), \quad v_{4}=(0,-1),
$$

and $\Sigma^{\prime}$ the complete fan spanned by

$$
v_{1}^{\prime}=(1,0), \quad v_{2}^{\prime}=(0,1), \quad v_{3}^{\prime}=(-1,1), \quad v_{4}^{\prime}=(-1,-1) .
$$

We will show that the rational cohomology algebras of the associated toric varieties $X_{\Sigma}$ and $X_{\Sigma^{\prime}}$ are not isomorphic. Straightforward simplifications of the algebra presentations according to Danilov (compare Theorem 2.1) yield the following descriptions:

$$
\begin{aligned}
& H^{*}\left(X_{\Sigma}, \mathbb{Q}\right) \cong \mathbb{Q}\left[u_{1}, u_{2}\right] /\left\langle u_{1} u_{2}, u_{1}^{2}+u_{2}^{2}\right\rangle \\
& H^{*}\left(X_{\Sigma^{\prime}}, \mathbb{Q}\right) \cong \mathbb{Q}\left[u_{1}, u_{2}\right] /\left\langle u_{1} u_{2}, u_{1}^{2}+2 u_{2}^{2}\right\rangle
\end{aligned}
$$

We compare nontrivial solutions for the system of equations

$$
\begin{aligned}
x^{2}=-y^{2} & =z, \\
x y & =0 .
\end{aligned}
$$

In $H^{*}\left(X_{\Sigma}, \mathbb{Q}\right),(x, y, z)=\left(u_{1}, u_{2}, u_{1}^{2}\right)$ obviously solves (3.4). Now assume that $x, y, z$ with $x=\alpha u_{1}+\beta u_{2}$ and $y=\gamma u_{1}+\delta u_{2}$ is a solution of (3.4) in $H^{*}\left(X_{\Sigma^{\prime}}, \mathbb{Q}\right)$. W.l.o.g. we can assume that the coefficients $\alpha, \beta, \gamma, \delta$ are integers with $\operatorname{gcd}(\alpha, \beta, \gamma, \delta)=1$. From $x y=0$ and $x^{2}=-y^{2}$ we deduce that

$$
\begin{aligned}
\beta \delta & =2 \alpha \gamma, \\
\beta^{2}+\delta^{2} & =2\left(\alpha^{2}+\gamma^{2}\right) .
\end{aligned}
$$

But these conditions imply that 2 is a common divisor of the coefficients, contradicting our assumption. Hence, the system of equations (3.4) has no non-trivial solution in $H^{*}\left(X_{\Sigma^{\prime}}, \mathbb{Q}\right)$ which shows that the algebras are not isomorphic.

We have thus proven the following:

Theorem 3.9. The rational cohomology algebra of a compact quasi-smooth toric variety is not a combinatorial invariant, not even in the projective case.

\section{COHOMOlOGY OF QUASI-SMOOTH TORIC VARIETIES IS NOT COMBINATORIAL}

We close our investigations with an example showing that already in complex dimension 3 neither the real nor the complex cohomology algebras of quasi-smooth toric varieties are combinatorial invariants.

Example 4.1. Consider the regular prism $P$ in $\mathbb{R}^{3}$ spanned by

$$
\begin{aligned}
& v_{1}=(1,0,-1), \quad v_{2}=(-1,1,-1), \quad v_{3}=(-1,-1,-1), \\
& v_{4}=(1,0,1), \quad v_{5}=(-1,1,1), \quad v_{6}=(-1,-1,1),
\end{aligned}
$$

and the triangulation of its boundary complex by the simplices

$$
\begin{array}{llll}
\left\{v_{1}, v_{2}, v_{3}\right\}, & \left\{v_{1}, v_{4}, v_{5}\right\}, & \left\{v_{1}, v_{2}, v_{5}\right\}, & \left\{v_{2}, v_{5}, v_{6}\right\}, \\
\left\{v_{2}, v_{3}, v_{6}\right\}, & \left\{v_{3}, v_{4}, v_{6}\right\}, & \left\{v_{1}, v_{3}, v_{4}\right\}, & \left\{v_{4}, v_{5}, v_{6}\right\} .
\end{array}
$$

Let $\Sigma$ denote the fan spanned by the triangulated boundary complex of $P$ over $0 \in \operatorname{int} P$. 
Define the polytope $P^{\prime}$ as the convex hull of

$$
\begin{aligned}
& v_{1}^{\prime}=(1,0,-1), \quad v_{2}^{\prime}=(-1,1,-1), \quad v_{3}^{\prime}=(-1,-1,-1), \\
& v_{4}^{\prime}=(1,-1,1), \quad v_{5}^{\prime}=(1,1,1), \quad v_{6}^{\prime}=(-1,0,1),
\end{aligned}
$$

and let $\Sigma^{\prime}$ denote the fan spanned by its boundary complex over $0 \in \operatorname{int} P^{\prime}$.

When "rotating" the top facet of $P$ clockwise, the maximal simplices in the above-mentioned triangulation of the boundary of $P$ are realized as facets of a convex polytope. The combinatorial type of $P$, i.e., its face lattice, coincides with that of $P^{\prime}$ : Both fans $\Sigma$ and $\Sigma^{\prime}$ have the combinatorial type of a fan spanned by an octahedron in $\mathbb{R}^{3}$. However, $\Sigma^{\prime}$ gives rise to a projective, $\Sigma$ to a non-projective toric variety.
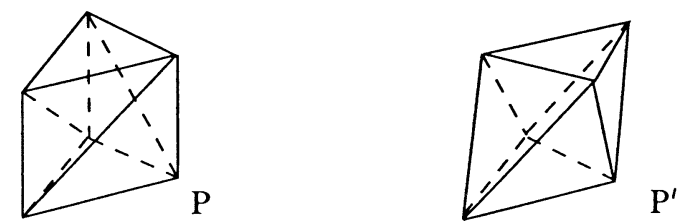

We will show that the cohomology algebras of the associated toric varieties $X_{\Sigma}$ and $X_{\Sigma^{\prime}}$ are not isomorphic as graded-commutative algebras, neither over $\mathbb{Q}$ nor over $\mathbb{R}$ or $\mathbb{C}$.

Setting $z_{1}=z_{5}+z_{6}, z_{2}=\frac{1}{2} z_{4}-\frac{1}{2} z_{5}+\frac{1}{2} z_{6}$, and $z_{3}=\frac{1}{2} z_{4}+\frac{1}{2} z_{5}-\frac{1}{2} z_{6}$ in the Danilov presentation for $H^{*}\left(X_{\Sigma}, \mathbb{K}\right)$, and setting $z_{1}=z_{6}, z_{2}=z_{4}$ and $z_{3}=z_{5}$ in the Danilov presentation for $H^{*}\left(X_{\Sigma^{\prime}}, \mathbb{K}\right), \mathbb{K}=\mathbb{Q}, \mathbb{R}$ or $\mathbb{C}$, we obtain the following algebra presentations:

$$
\begin{aligned}
H^{*}\left(X_{\Sigma}, \mathbb{K}\right) \cong \mathbb{K}\left[z_{4}, z_{5}, z_{6}\right] /\left\langle\begin{array}{l}
z_{4} z_{5}+z_{5}^{2}+z_{6}^{2}, \\
z_{4} z_{6}+z_{4}^{2}+z_{5}^{2}+z_{6}^{2}, \\
z_{5} z_{6}+z_{6}^{2}
\end{array}\right\rangle, \\
H^{*}\left(X_{\Sigma^{\prime}}, \mathbb{K}\right) \cong \mathbb{K}\left[z_{4}, z_{5}, z_{6}\right] /\left\langle z_{4}^{2}, z_{5}^{2}, z_{6}^{2}\right\rangle .
\end{aligned}
$$

We show that all squares of non-zero elements from $H^{2}\left(X_{\Sigma}, \mathbb{K}\right)$ are non-zero: Expanding the square of an arbitrary element $\lambda z_{4}+\mu z_{5}+\nu z_{6}$ of $H^{2}\left(X_{\Sigma}, \mathbb{K}\right)$, $\lambda, \mu, \nu \in \mathbb{K}$, in the linear basis $z_{4}^{2}, z_{5}^{2}, z_{6}^{2}$ of $H^{4}\left(X_{\Sigma}, \mathbb{K}\right)$, and assuming that the square is 0 , we obtain the following system of equations in the coefficients $\lambda, \mu, \nu$ :

$$
\begin{aligned}
& 0=\lambda^{2}-2 \lambda \nu, \\
& 0=\mu^{2}-2 \lambda \nu-2 \lambda \mu, \\
& 0=\nu^{2}-2 \lambda \mu-2 \lambda \nu-2 \mu \nu .
\end{aligned}
$$

In neither of the considered coefficient fields this system of equations has a solution other than the trivial one; hence squares of elements in $H^{2}\left(X_{\Sigma}, \mathbb{K}\right)$ are non-zero.

However, the defining relations in the before-mentioned presentation of $H^{*}\left(X_{\Sigma^{\prime}}, \mathbb{K}\right)$ already show that there are elements in $H^{2}\left(X_{\Sigma^{\prime}}, \mathbb{K}\right)$ whose squares are zero. We conclude that the algebras are non-isomorphic, and have thus proven the following:

Theorem 4.2. For compact quasi-smooth toric varieties in general, neither the rational nor the real or complex cohomology algebras are determined by the combinatorial data of the defining fans. 


\section{ACKNOWLEDGMENTS}

I thank Mark Goresky, Mark McConnell and Günter M. Ziegler for helpful comments, and, in particular, Dmitry Kozlov for critical questions and steady discussions along all stages of this work. Also, I thank the referee for the careful reading that he gave to this article, and for his suggestions that led to improve the exposition of the material.

\section{REFERENCES}

[Bi] L. Billera: The algebra of continuous piecewise polynomial functions; Adv. Math. 76 (1989), 170-183. MR 90g:13021

[Br] M. Brion: The structure of the polytope algebra; Tôhoku Math. J. 49 (1997), 1-32. MR 98a:52019

[Da] V.I. Danilov: The geometry of toric varieties; Russ. Math. Surv. 33 (1978), 97-154. MR 80g:14001

[Ew] G. Ewald: Combinatorial Convexity and Algebraic Geometry; Graduate Texts in Mathematics 168, Springer-Verlag, 1996. MR 97i:52012

[FY] S. Fischli, D. Yavin: Which 4-manifolds are toric varieties?; Math. Z. 215 (1994), 179185. MR 95g:57045

[Fu] W. Fulton: Introduction to Toric Varieties; Annals of Mathematics Studies 131, Princeton University Press, 1993. MR 94g:14028

[GM] M. Goresky, R. MacPherson: Intersection homology theory; Topology 19 (1980), 135-162. MR 82b:57010

[GH] P. Griffiths, J. Harris: Principles of Algebraic Geometry; Wiley-Interscience, New York, 1978. MR 80b:14001

[McC] M. McConnell: The rational homology of toric varieties is not a combinatorial invariant; Proc. Amer. Math. Soc. 105 (1989), 986-991. MR 89i:14042

[McM] P. McMullen: On simple polytopes; Invent. Math. 113 (1993), 419-444. MR 94d:52015

[Od] T. Oda: Convex Bodies and Algebraic Geometry; Ergebnisse der Mathematik und ihrer Grenzgebiete, 3. Folge, Bd. 15, Springer-Verlag, 1988. MR 88m:14038

[Sa] M. Saito: Hodge structures via filtered D-modules; Astérisque 130 (1985), 342-351. MR 87b:32019

[Zi] G.M. Ziegler: Lectures on Polytopes; Graduate Texts in Mathematics 152, SpringerVerlag, 1995. MR 96a:52011

Department of Mathematics, ETH Zurich, 8092 Zurich, Switzerland

E-mail address: feichtne@math.ethz.ch 